\title{
Surgical repair of a quadricuspid aortic valve associated with a ruptured sinus of Valsalva aneurysm
}

\author{
Ming $\mathrm{Xu}, \mathrm{MD}$, Laichun Song, MD, Liang Tao, $\mathrm{PhD}$, and Hongyan Xiao, MD
}

\author{
From the Department of Cardiac Surgery, Wuhan Asia Heart Hospital, Wuhan, People's Republic of China. \\ This work was supported by the 2017 Wuhan Young and Middle-Aged Medical Backbone Training Program; the \\ Health and Family Planning Commission of Wuhan municipality scientific research project (WX17Q35); and \\ the Wuhan Science and Technology Bureau (2016060101010036). \\ Drs Song, $\mathrm{Xu}$, and Xiao contributed equally to the article. \\ Disclosures: Authors have nothing to disclose with regard to commercial support. \\ Received for publication May 29, 2019; revisions received June 23, 2019; accepted for publication June 27, 2019; \\ available ahead of print Aug 10, 2019. \\ Address for reprints: Liang Tao, PhD, Department of Cardiac Surgery, Wuhan Asia Heart Hospital, No.753 Jing- \\ han Ave, Hankou District, Wuhan 430022, People's Republic of China (E-mail: taoliangmd@sina.com). \\ J Thorac Cardiovasc Surg 2020;159:e341-2 \\ $0022-5223 / \$ 36.00$ \\ Copyright (C) 2019 by The American Association for Thoracic Surgery \\ https://doi.org/10.1016/j.jtcvs.2019.06.085
}

A 35-year-old woman presented with a history of gradually worsening dyspnea on exertion. Clinical examination revealed a diastolic murmur heard loudest along the upper left sternal border, which did not radiate to the carotids or the precordium. Transthoracic echocardiography demonstrated a quadricuspid aortic valve (type A, Hurwitz and Robert's classification) with an aorta-to-right atrium fistula (sinus 2) through a ruptured right-anterior sinus of Valsalva (Figure 1, $A$ ). In addition, the coronary orifices originated from sinus 1 (right coronary artery) and sinus 4 (left main coronary artery), and the aortic regurgitation was moderate (Figure 1, $A$ and $B$ ). A diagnosis of a congenital quadricuspid aortic valve associated with ruptured sinus of Valsalva aneurysm was made, and the patient was

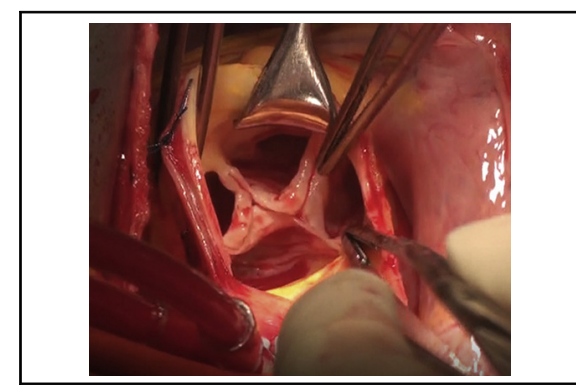

Quadricuspid aortic valve associated with ruptured sinus of Valsalva aneurysm.

Central Message

The quadricuspid aortic valve associated with ruptured sinus of Valsalva aneurysm is rare; however, we report successful repair of both abnormalities.

See Article page e343.

referred for surgical correction. During the procedure, the aortic valve was confirmed to have 4 leaflets with associated sinuses, and a ruptured right-anterior sinus of Valsalva without coronary orifices was identified (Figure 2, A). The proximal windsock orifice was below the right-anterior sinus of Valsalva, and a right angle forceps could easily be passed through the aorta-to-right atrium fistula into the right atrium (Figure 2, B). The ruptured sinus of Valsalva

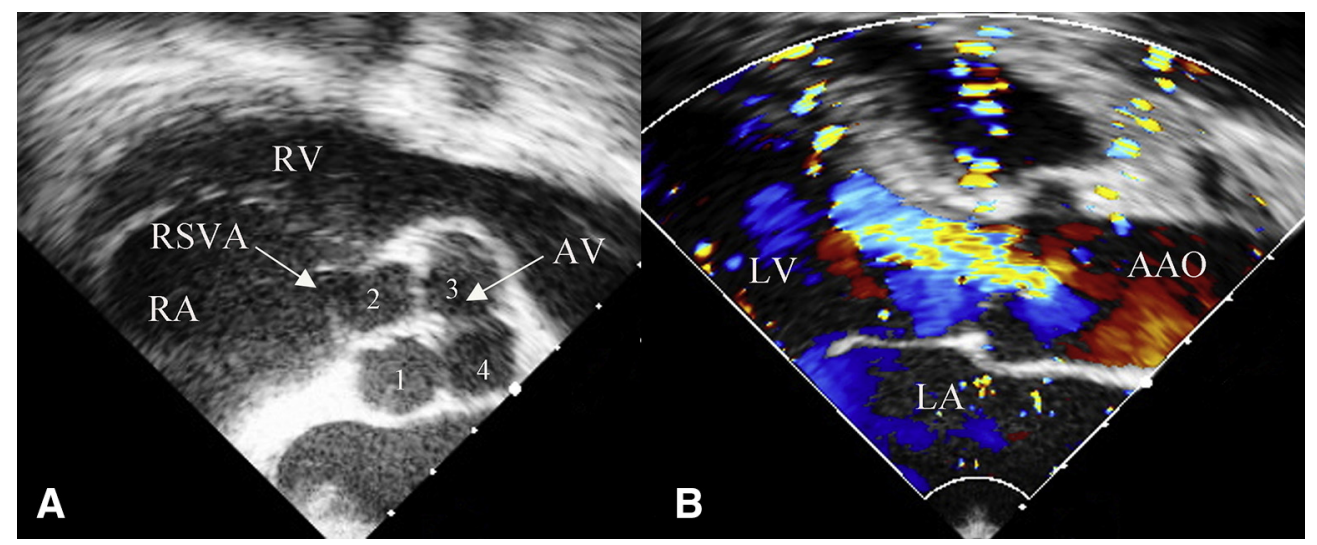

FIGURE 1. A, Transthoracic echocardiography demonstrated a quadricuspid aortic valve with 4 sinuses (right coronary artery in sinus 1 ; left main artery in sinus 4) and a ruptured right-anterior sinus of Valsalva. B, Transthoracic echocardiography showed moderate aortic regurgitation. $R V$, Right ventricle; $R S V A$, right sinus of Valsalva aneurysm; $R A$, right atrium; $A V$, aortic valve; $L V$, left ventricle; $L A$, left atrium; $A A O$, ascending aorta. 


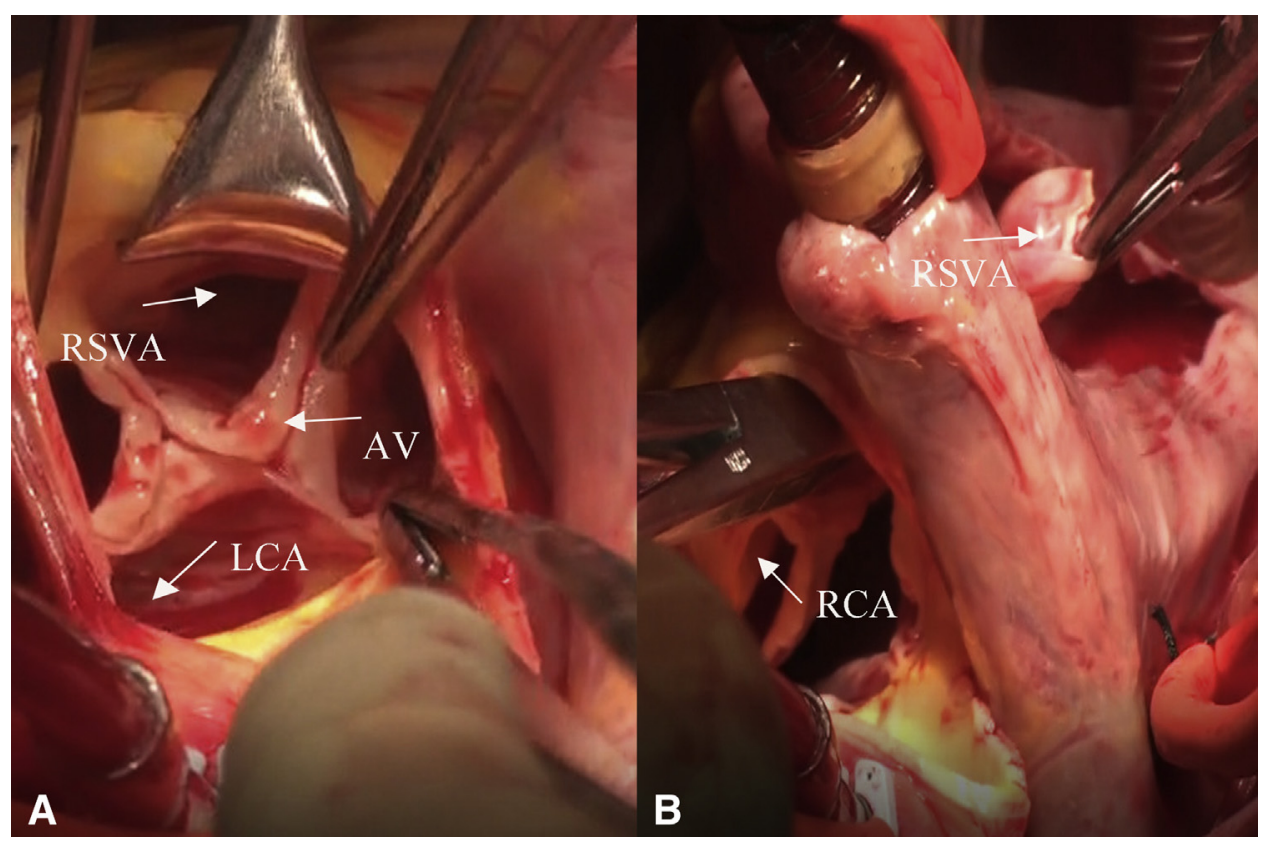

FIGURE 2. A and B, During the procedure, the quadricuspid aortic valve was confirmed with 4 sinuses; a right angle forceps was used to identify the aortato-right atrium fistula. $R S V A$, right sinus of Valsalva aneurysm; $A V$, aortic valve; $L C A$, left coronary artery; $R C A$, right coronary artery.

aneurysm with an associated leaflet was resected. A 40 polypropylene suture was used to reconstruct the aortic root with 3 leaflets and associated sinuses. Postoperative transesophageal echocardiography showed normal aortic valvular function with no aortic insufficiency or residual shunt (Figure 3, $A$ and $B$ ). The patient recovered well and was discharged a few days later, with no further complications.

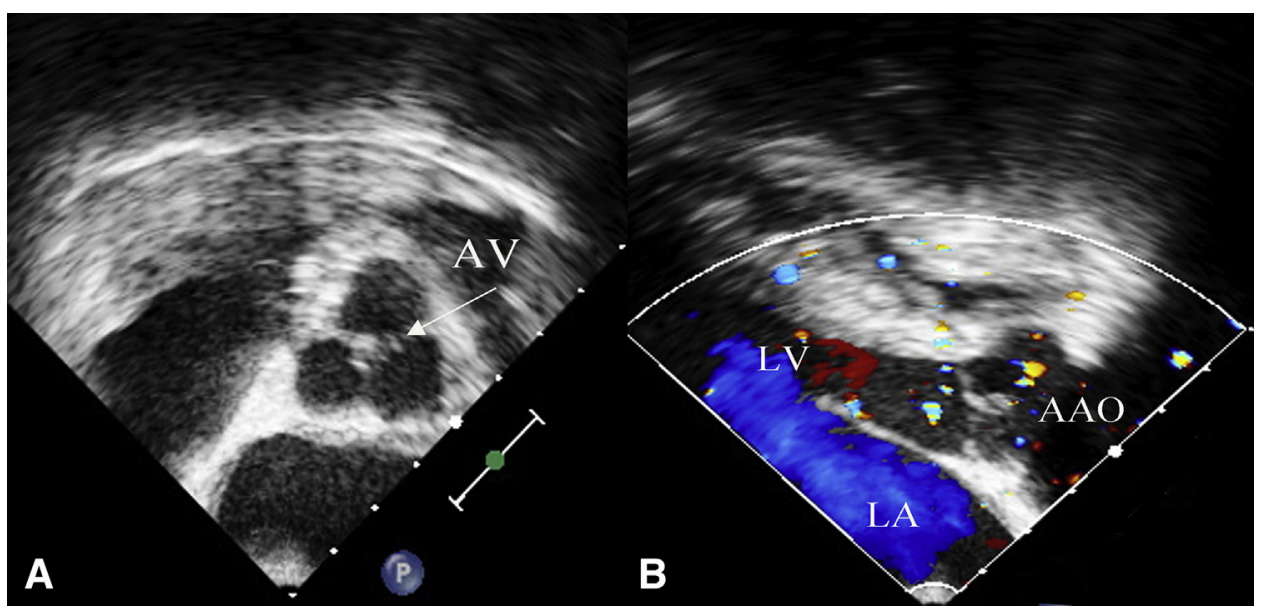

FIGURE 3. A and B, Postoperative transesophageal echocardiogram showed a normal aortic valve with 3 cusps and no aortic insufficiency or residual shunt. $A V$, aortic valve; $L V$, left ventricle; $A A O$, ascending aorta; $L A$, left atrium. 